\title{
STABILITY FOR INHOMOGENEOUS DIFFERENCE SCHEMES ${ }^{1}$
}

T. SEIDMAN

PART I

Preliminaries. We consider a PDE

$$
u_{t}-A u=f
$$

where $u$ is a (possibly vector-valued) unknown function of a real "time" variable $t$ and an $N$-dimensional real vector "space" variable $x$. Here $A$ is a linear operator, constant ${ }^{2}$ in $t$, operating on $u$, where $u$ is considered a function of $x$ alone (i.e., $A$ acts on elements of a linear space $B$ and, for each value of $t, u(\cdot, t) \in B)$. The function $f$ is a known function of $x$ and $t$. Thus $u$ is to satisfy (1) for $t>0$ and $x \in R$. The initial condition

$$
u(x, 0)=\bar{u}(x) \quad(x \in R)
$$

and the boundary condition

$$
\Gamma u(\cdot, t)=g(t)=g(*, t)
$$

are assumed to hold. The operator $\Gamma$ is a linear transformation from $B$ to another linear space $D$ of functions $[g(t) \in D$ for each $t>0]$ defined on $\partial R$, the boundary of $R$, such that $\Gamma u$ depends only on the values of $u$ near the boundary.

We assume this problem is well-posed. That is, we assume the existence, for each fixed $T>0$, of a one-parameter family of operators $\hat{S}_{t}$ acting on a space $\Sigma$ with elements of the form

$$
w=\left(\begin{array}{l}
\bar{u} \\
\gamma \\
\phi
\end{array}\right),
$$

where $\bar{u}$ is a function ${ }^{3}$ of $x \in R, \gamma$ is a function of $x \in \partial Q$ and $0<t$

Received by the editors September 21, 1960. mission.

1 This work was performed under the auspices of the U. S. Atomic Energy Com-

${ }^{2}$ As in [1], this is not a necessary requirement but is imposed only to avoid notational clumsiness. The modification necessary to the general case should be clear. The operator $\Gamma$ appearing in equation (3) may also depend on $t$.

${ }^{3}$ The values of $\bar{u}$ and $\phi$ will be the same sort of vectors as $u$ and $f_{i}$ those of $\gamma$ will be as $g$. 
$\leqq T$, and $\phi$ is a function of $x \in R$ and $0<t \leqq T$. We also assume that each $w \in \Sigma$ satisfies

$$
\Gamma \bar{u}=\gamma(0) .
$$

The operators $\hat{S}_{t}$ are to send $\Sigma$ into $\leftrightarrow$ in such a way that for each $w \in \Sigma$
(a) $\left\|\hat{S}_{t} w-\bar{u}\right\| \rightarrow 0$,
as $t \rightarrow 0+$,
(b) $\Gamma \hat{S}_{t} w=\gamma(t)$ for $0<t \leqq T$,
(c) $\left\|\left(\hat{S}_{t+\delta} w-\hat{S}_{t} w\right) / \delta-A \hat{S}_{t} w-\phi(t)\right\| \rightarrow 0$, as $\delta \rightarrow 0$, for $0<t<T$,
for $0<t \leqq T$.
(d) $\left\|\hat{S}_{t}\right\| \leqq M=M(T)$,

It is convenient to define the translation operators ${ }^{4} T_{t}$ and $T_{t}$ by

$$
\begin{aligned}
& \tau+t \leqq T, x \in \partial R, \\
& \tau+t>T, x \in \partial R ; \\
& \tau+t \leqq T, x \in R, \\
& \tau+t>T, x \in R .
\end{aligned}
$$

Clearly, $\hat{S}_{t}$ can be written as

$$
\hat{S}_{\imath}=\left(U_{t}, G_{t}, F_{t}\right),
$$

where

$$
U_{t} \bar{u}=\hat{S}_{t}\left(\begin{array}{l}
\bar{u} \\
0 \\
0
\end{array}\right), \quad G_{t} \gamma=\hat{S}_{t}\left(\begin{array}{l}
0 \\
\gamma \\
0
\end{array}\right), \quad F_{t} \phi=\hat{S}_{t}\left(\begin{array}{l}
0 \\
0 \\
\phi
\end{array}\right),
$$

and it is convenient to define the semi-group $\left\{S_{t}\right\}$ by

$$
S_{t}=\left(\begin{array}{ccc}
U_{t} & G_{t} & F_{t} \\
0 & T_{t} & 0 \\
0 & 0 & T_{t}
\end{array}\right) .
$$

It follows from (5) that $\left\{S_{t}\right\}$ is a bounded, strongly continuous ${ }^{5}$ semigroup, and provides the solution of the problem determined by (1), (2), and (3).

4 There should be no confusion caused by using the same letter for these two different operators, distinguishing them only by their arguments.

- We assume that $\phi$ and $\gamma$ are restricted so that the operators $T_{t}$ are strongly continuous. For example, we may assume them to be continuous in $t$ if the maximum norm is used. Actually, a finite number of discontinuities may be handled also without difficulty. 
If we now consider, for each $\delta>0$, a mesh $\mu_{\delta}$ consisting of a finite number of points in $R$, each taken at $t=0, \delta, 2 \delta, \cdots$, then (sufficiently smooth) functions in $\Sigma$ may be approximated by functions determined by their values on $\mu_{\delta}$. We identify the approximating functions with functions on $\mu_{\delta}$ in such a way as to approximate norms.

By a difference scheme, we mean a family of operators $\widetilde{S}_{\delta}$ such that $\left(\tilde{S}_{\delta}\right)^{n}$ approximates $S_{n \delta}$ in an appropriate sense. We will write $\tilde{S}_{\delta}$ in the form

$$
\tilde{S}_{\delta}=\left(\begin{array}{ccc}
\tilde{u}_{\delta} & \tilde{G}_{\delta} & \tilde{F}_{\delta} \\
0 & \tilde{T}_{\delta} & 0 \\
0 & 0 & \tilde{T}_{\delta}
\end{array}\right),
$$

and, if $\tilde{w}$ is the function on $\mu_{\delta}$ corresponding to an arbitrary but sufficiently smooth function $w \in \Sigma$ of the type described above [let $\tilde{w}_{1}=\tilde{u}, \tilde{w}_{2}=\tilde{\gamma}, \tilde{w}_{3}=\tilde{\phi}$, where $\left.\tilde{w}=(\tilde{u}, \tilde{\gamma}, \tilde{\phi})^{T}\right]$, we will ask that

$$
\left\|\left[\left(\tilde{S}_{\delta} \tilde{w}\right)_{1}-\tilde{u}\right] / \delta-A u-\phi(0)\right\| \rightarrow 0 \quad \text { as } \delta \rightarrow 0 .
$$

If (10) is satisfied, we say that $\widetilde{S}_{\delta}$ is consistent with the problem. If there is a constant $\tilde{M}=\tilde{M}(T)$ such that

$$
|| \tilde{S}_{\delta^{n}}|| \leqq \tilde{M} \quad \text { for } 0 \leqq n \delta \leqq T,
$$

then we say the scheme is siable. If $\widetilde{S}_{\boldsymbol{\delta}}^{n}$ converges strongly to $S_{t}$ as $\delta \rightarrow 0$ with $(n \delta) \rightarrow t$, we say the scheme is convergent.

Convergence implies stability. The proof here is identical with that in $[1]$.

Suppose the scheme is convergent. Then, since $[0, T]$ is closed, either $\left\|\tilde{S}_{\delta}{ }^{n} w_{0}\right\|$ is uniformly bounded for each $w_{0} \in \Sigma$ and $n \delta \leqq T$, or, for some fixed $t_{0} \in[0, T]$ and fixed $w_{0} \in \Sigma$, there would be a sequence $\delta_{j} \rightarrow 0$ and a sequence $n_{j}$ such that $n_{j} \delta_{j} \rightarrow t_{0}$ for which $\left\|\left(S_{\delta_{j}}\right)^{n_{i}} \tilde{w}_{0}\right\|$ becomes arbitrarily large. This, however, would contradict the convergence; thus, for each $w_{0} \in \Sigma$ there is an $m=m\left(T, w_{0}\right)$ such that $\left\|\tilde{S}_{\delta}{ }^{n} w_{0}\right\| \leqq m$ for $0 \leqq n \delta \leqq T$. Since, for each $\delta, \tilde{S}_{\delta}$ is effectively finitedimensional, it is obviously bounded as is $\widetilde{S}_{8}{ }^{n}$. Hence, by the Principle of Uniform Boundedness, the operators $\tilde{S}_{8}{ }^{n}$ are uniformly bounded for $n \delta \leqq T$ and the scheme is stable.

Stability implies convergence. This again is substantially as presented in [1].

Let $w_{0} \in \Sigma$ and $w(t)=S_{t} w_{0}$. From (5) the "first component" of $w(t)$ is the desired solution of the problem. By (5c), we have, for any $\epsilon>0$, a $\delta_{1}$ such that, for $0<\delta<\delta_{1}$, 


$$
\left\|\left[\hat{S}_{\delta} w(t)-u(t)\right] / \delta-A u(t)-\phi(t)\right\| \leqq \epsilon / 4 \tilde{M} T \quad \text { for } 0 \leqq t<T .
$$

Assuming the scheme $\left\{\tilde{S}_{\delta}\right\}$ is stable, we have by (10), with $w=w(t)$, a $\delta_{2}$ such that, for $0<\delta<\delta_{2}$,

(13) $\left\|\left[\left(\tilde{S}_{8} \tilde{w}(t)\right)_{1}-\tilde{u}(t)\right] / \delta-A u(t)-\phi(t)\right\| \leqq \epsilon / 4 \tilde{M} T$ for $0 \leqq t<T$.

Note further that for $w$ sufficiently smooth there is a $\delta_{3}$ such that for $0<\delta<\delta_{3}$

$$
\|\tilde{u}-u\| \leqq \epsilon / 4 \tilde{M} T \quad \text { for } 0 \leqq t \leqq T .
$$

It follows from (11), (12), and (13) that for $0<\delta<\delta_{1}, \delta_{2}, \delta_{3}$

$$
\left\|\left(\tilde{S}_{\delta} \tilde{w}(t)\right)_{1}-\hat{S}_{\delta} w(t)\right\| \leqq 3 \epsilon \delta / 4 \tilde{M} T \quad \text { for } 0 \leqq t \leqq T .
$$

Let $\delta_{j} \rightarrow 0+$, and $n_{j}$ be such that $n_{j} \delta_{j} \rightarrow t \in[0, T]$. Set

$$
\begin{aligned}
\psi_{j} & =\left[\left(\tilde{S}_{\delta_{j}}\right)^{n_{i} \tilde{w}_{0}}\right]_{1}-\hat{S}_{n_{j} \delta_{j}} w_{0} \\
& =\sum_{k=0}^{n_{j}-1}\left[\tilde{S}_{\delta_{j}}^{k}\left(\widetilde{S}_{\delta_{j}}-S_{\delta_{j}}\right) S_{\left(n_{j}-1-k\right) \delta_{j}} w_{0}\right]_{1} .
\end{aligned}
$$

Then, using the stability condition and (15), with $t=\left(n_{j}-1-k\right) \delta_{j}$, we have, for $\delta_{j}<\delta_{0}=\min \left\{\delta_{1}, \delta_{2}, \delta_{3}\right\}$,

$$
\left\|\psi_{j}\right\| \leqq \sum_{0}^{n_{j}-1} \tilde{M}\left(3 \epsilon \delta_{j} / 4 \tilde{M} T\right)=\frac{3}{4}\left(n_{j} \delta_{j} / T\right) \epsilon \leqq \frac{3}{4} \epsilon .
$$

Putting $s=\left|n_{j} \delta_{j}-t\right|$, we have

$$
\pm\left(S_{n_{j} \delta_{j}}-S_{t}\right) w_{0}=S_{t^{\prime}}\left(S_{s}-I\right) w_{0},
$$

where the sign on the left denotes whether $n_{j} \delta_{j}$ is greater than or less than $t$, and where $t^{\prime}$ is the smaller of $n_{j} \delta_{j}$ and $t$. Therefore,

$$
\left\|\hat{S}_{n_{j} \delta_{j}} w_{0}-\hat{S}_{t} w_{0}\right\| \leqq\left\|S_{t^{\prime}}\right\| \cdot\left\|S_{s} w_{0}-w_{0}\right\| \text {, }
$$

which goes to zero as $s \rightarrow 0$ (i.e., as $j \rightarrow \infty$ ), according to (5d) and (5a). Thus, for any $\epsilon>0$, there is a $J_{1}$ such that for $j>J_{1}$

$$
\left\|\hat{S}_{n_{j} \delta_{j}} w_{0}-\hat{S}_{t} w_{0}\right\| \leqq \epsilon / 4 .
$$

Letting $J$ be greater than $J_{1}$ and also large enough so that $\delta_{j}<\delta_{0}$ for $j>J$, we have, then, from (15), (17), and (19),

$$
\begin{aligned}
\left\|\left(\tilde{S}_{\delta_{j}}^{n_{j}} \tilde{w}_{0}\right)_{1}-\hat{S}_{t} w_{0}\right\| & =\left\|\psi_{j}+\hat{S}_{n_{j} \delta_{j}} w_{0}-\hat{S}_{t} w_{0}\right\| \\
& \leqq 3 \epsilon / 4+\epsilon / 4=\epsilon .
\end{aligned}
$$

Thus, since $\epsilon$ was arbitrary, we have shown that $\left(\widetilde{S}_{\delta_{j}}\right)^{n_{j}}$ converges 
strongly to $S_{t}$ as $j \rightarrow \infty$, and, since the sequence $\left\{\delta_{j}\right\}$ was arbitrary, this proves convergence.

\section{PART II}

The concept of stability can be generalized to boundary value problems of the form:

$$
\begin{aligned}
& L u=f, \\
& \Gamma u=\gamma,
\end{aligned}
$$

where $L$ is a linear differential operator acting on functions $u=u(x)$ for $x$ in a domain $D$ and $\Gamma$ is a linear boundary operator mapping $u$ into a function $\boldsymbol{\gamma}$ defined on $\partial D$.

If the problem is well-posed, there is a bounded linear operator $S$ such that

$$
S\left(\begin{array}{l}
f \\
\gamma
\end{array}\right)=u
$$

gives the solution $u$ of (21). Clearly $S$ is the inverse of

$$
A=\left(\begin{array}{l}
L \\
\Gamma
\end{array}\right) .
$$

By a difference scheme we mean a parametrized sequence of meshes $\mu_{\delta}$ becoming dense as $\delta \rightarrow 0$ and a family of operators $\widetilde{S}_{\delta}$ intended $^{6}$ to approximate $S$ as $\delta \rightarrow 0$.

We say the scheme $\left\{\tilde{S}_{\delta}\right\}$ is consistent with (21) if for every sufficiently smooth function $u$

$$
\left\|A u-\tilde{S}_{\delta}^{-1} \tilde{u}\right\| \rightarrow 0 \quad \text { as } \delta \rightarrow 0 .
$$

The scheme is called stable if the operators $\widetilde{S}_{\delta}$ are uniformly bounded so there is a constant $M$ such that

$$
\left\|\tilde{S}_{\delta} \tilde{w}\right\| \leqq M\|\tilde{w}\| \leqq M^{\prime}(\|f\|+\|\gamma\|) \text {. }
$$

Finally, the scheme is convergent if $\tilde{S}_{\delta}$ converges strongly to $S$ as $\delta \rightarrow 0$ so that for a dense set of $w$

$$
\left\|\tilde{S}_{\delta} \tilde{w}-S w\right\| \rightarrow 0 \quad \text { as } \delta \rightarrow 0 .
$$

H. Keller observed ${ }^{7}$ that it is extremely simple to show that stability implies convergence in this situation also. Ignoring the distinction

${ }^{6}$ In practice, of course, it is $\widetilde{S}_{\delta}^{-1}$ which is given.

${ }^{7}$ Private communication. This will appear in the forthcoming book on numerical analysis by $\mathrm{E}$. Isaacson and $\mathrm{H}$. Keller. 
between such functions as $u$ and $\tilde{u}$ (the representative function agreeing with $u$ on $\mu_{\delta}$ and approaching $u$ in the norm) the proof runs as follows:

From (23), there is, for any $\epsilon>0$, a $\delta_{0}$ such that, for $\delta<\delta_{0}$, $\left\|w-S_{\delta}^{-1} u\right\|<\epsilon$ where we write $A u=w, S w=u$. On multiplying $w^{\prime}=\left(w-\tilde{S}_{\delta}^{-1} u\right)$ by $\tilde{S}_{\delta}$ one has, from (24) $\left\|\tilde{S}_{\delta} w-u\right\|=\left\|S_{\delta} w^{\prime}\right\| \leqq M^{\prime}\left\|w^{\prime}\right\|$ $<M^{\prime} \epsilon$ which gives (25) and the scheme is convergent.

As in Part I, the converse follows from the Principle of Uniform Boundedness.

\section{REFERENCE}

1. P. Lax and R. Richtmeyer, Survey of the stability of linear finite difference equations, Comm. Pure Appl. Math. vol. 9 (1956) pp. 267-293.

University of CALIFornia, Livermore

\section{DUALITY IN HOMOGENEOUS PROGRAMMING ${ }^{1}$}

\section{E. EISENBERG}

The problem of maximizing a concave function subject to linear constraints does not have a dual, as is the case in linear programming, in which primal optimizing variables do not appear. As a special case of our principal result it will follow that such a dual does indeed exist whenever the objective function is also homogeneous.

In the linear case we are given an $m \times n$ matrix $A$ and vectors $a \in R^{n}, b \in R^{m} .^{2}$ The feasibility sets $X$ and $Y$ are defined by: $X=R_{+}^{m} \cap\{x \mid x A \leqq a\}, Y=R_{+}^{n} \cap\{y \mid A y \geqq b\}$. Since $x A \leqq a$ if and only if $x A y \leqq a y$ for all $y \in R_{+}^{n}$ (and similarly for $A y \geqq b$ ), we may write:

$$
\begin{aligned}
& X=R_{+}^{m} \cap\left\{x \mid x A y \leqq \psi(y) \quad \text { all } y \in R_{+}^{n}\right\} \\
& Y=R_{+}^{n} \cap\left\{y \mid x A y \geqq \phi(x) \quad \text { all } x \in R_{+}^{m}\right\}
\end{aligned}
$$

Received by the editors April 26, 1960; and in revised form, July 27, 1960 and September 12, 1960.

${ }^{1}$ Work on this paper was supported, in part, by the Logistics Branch of the Office of Naval Research under Contract NONR 562(15) at Brown University.

${ }^{2} R^{m}$ denotes the set of all real $m$-tuples. If $u, v \in R^{m}$ then $u \leqq v$ means that the inequality holds for each component. In particular, $R_{+}^{m}=R^{m} \bigcap\{x \mid x \geqq 0\}$. If $M$ is a $p \times q$ matrix and $N$ is a $q \times t$ matrix then $M N$ represents the usual matrix product. To simplify notation, the same symbol is used for both a column vector and its transpose; the meaning will, in any case, be clear from the context. 